\title{
Uso de Realidad Virtual y Realidad Aumentada en el Arbitraje Internacional
}

\author{
Ricardo Vergara Olmos*
}

\section{Resumen}

El avance tecnológico ha traído consigo múltiples cambios en la manera en que se desarrolla el proceso arbitral. En este artículo se analiza el impacto que genera el uso de realidad virtual y realidad aumentada en la producción y ponderación de la prueba, revisando tanto los beneficios como los posibles problemas de su implementación. Entre estos últimos, se profundiza en la alteración del rol del árbitro debido al efecto inmersivo de la realidad virtual, en el reemplazo del juzgador al momento de interpretar los antecedentes sobre los que se basa el diseño de la simulación y en los problemas de costos de utilización en el arbitraje internacional. Finalmente, se realizan una serie de recomendaciones en aras de favorecer una implementación cuidadosa de esta tecnología en el proceso arbitral.

Palabras clave: Arbitraje internacional - Realidad virtual - Realidad aumentada.

\begin{abstract}
The technological development has brought many changes in the way that the arbitral process takes place. This article analyzes the impact generated by the use of virtual reality and augmented reality for the production and evaluation of evidence, examining both the benefits and the possible problems that may arise by the implementation of such technology. Within its problems, this article deepens in the alteration of the arbitrator's role caused by the immersive effect of virtual reality, in the replacement of the judge regarding the interpretation of the fact upon which the simulation is based, and in the costs of using these technologies in international arbitration. Finally, this article gives some recommendations in order to provide for a careful implementation of this technology in the arbitral process.
\end{abstract}

Keywords: International Arbitration - Virtual Reality - Augmented Reality.

\footnotetext{
* Egresado de la Facultad de Derecho de la Universidad de Chile. Ayudante de Derecho Civil y Derecho Comercial en la misma casa de estudios. Entrenador del Equipo de la Universidad de Chile para The Annual Willem C. Vis International Commercial Arbitration Moot. Email: ricardo.vergaraolmos@gmail.com. Recibido: 11 de noviembre, 2019; modificaciones: 15 de agosto, 2020; aceptado: 18 de agosto, 2020.
} 
Ricardo Vergara Olmos

Uso de Realidad Virtual y Realidad Aumentada en el Arbitraje Internacional

\section{Introducción}

El avance tecnológico ha cambiado la forma en que se desarrolla la actividad jurídica. Esta variación en la práctica del derecho se verifica, entre otros ámbitos, en la litigación arbitral internacional. Precisamente, el uso de videoconferencias, el empleo de aplicaciones electrónicas para traducir conversaciones en vivo y los mecanismos de almacenamiento digital de datos han favorecido la inmediación, la comunicación y la disminución de costos en el arbitraje internacional (Wahab \& Katsh, 2018).

Pero eso no es todo. Las nuevas tecnologías también han generado grandes cambios metodológicos al momento de presentar un caso persuasivamente ante un tribunal arbitral. Uno de los mayores ejemplos de ello es la utilización de realidad virtual y realidad aumentada en el arbitraje, ya que no solo permite acercar al árbitro a una realidad a la que no tuvo acceso al momento de su ocurrencia, sino que además permite, por medios didácticos, dar cuenta de la forma en que se desarrollaron los hechos alegados.

Como aporte a la labor de comprensión y determinación de los hechos del tribunal arbitral, el uso de estas tecnologías significa en principio un gran avance. Sin embargo, su empleo supone también una serie de riesgos para el recto cumplimiento del deber de juzgamiento de los árbitros. Ello se debe tanto a la forma en que se le presenta la información al juzgador, como también a la manera en que inciden las partes y los diseñadores de la simulación respectiva en la manifestación de los hechos alegados, supuestamente existentes en el modo en el cual se presenta el material audiovisual por las partes al tribunal arbitral.

La falta de desarrollo en esta materia genera una serie de interrogantes cuya respuesta resulta fundamental para la eventual extensión del uso de esta tecnología. ¿Qué son concretamente la realidad virtual y la realidad aumentada? ¿Cuáles son sus beneficios para el proceso arbitral? ¿Qué peligros entraña su aplicación para el debido proceso? ¿Qué podemos hacer para enfrentar esos peligros? Y, por supuesto, ¿vale la pena el uso de esta tecnología, atendidas sus posibles dificultades?

Con base en las preguntas anteriores, este artículo tiene por objetivo analizar el uso de la realidad virtual y la realidad aumentada en el arbitraje internacional, identificando los aspectos problemáticos de su implementación y proveyendo criterios y recomendaciones para un uso prudente y seguro de estas herramientas. Esto demostrando que, a pesar de las inquietudes que su desconocimiento pueda generar, la realidad virtual y la realidad aumentada son un aporte y una oportunidad para mejorar la calidad de la litigación arbitral.

Para ello, se ha revisado literatura especializada en la tecnología en cuestión, sus características y sus funciones, con el fin de ofrecer un panorama conceptual y técnico adecuado a nuestros objetivos. También se ha recurrido a doctrina autorizada en arbitraje, tanto en cuanto a las instituciones y principios que informan el proceso arbitral, como al estado actual de la práctica en el área. Por último, se ha mantenido un enfoque centrado en la litigación, prestando especial atención al carácter persuasivo del modo de presentar la evidencia al tribunal arbitral.

En cuanto a la estructura de la presente investigación, comenzaremos por definir la realidad virtual y la realidad aumentada, dando cuenta de sus características y beneficios. Luego, explicaremos su relevancia como instrumentos de prueba y persuasión en el arbitraje internacional, para después identificar, describir 
y reflexionar sobre las cuestiones problemáticas del empleo de esta tecnología. Determinados los aspectos problemáticos, procederemos a efectuar algunas observaciones y recomendaciones para un uso adecuado de la realidad virtual y la realidad aumentada en el arbitraje, finalizando con unas breves conclusiones.

\section{Marco conceptual: realidad virtual y realidad aumentada}

La realidad virtual es definida por la Real Academia Española como la representación de escenas o imágenes de objetos producida por un sistema informático, que da la sensación de su existencia real. Sin embargo, se ha resaltado por algunos autores que la realidad virtual apunta a la estimulación de uno o más sentidos, por lo que resulta ser más bien un producto informático que interactúa con la persona como un todo más que como un mero espectador (Brudniy \& Demilhanova, 2012). De ahí que la International Bar Association (IBA) enfatice que la realidad virtual tiene un efecto de inmersión o de estimulación completa de los sentidos, al decir que esta tecnología "allows the creation of immersive virtual environments (IVE), which are artificial, interactive, computer-created scenes combining highresolution projections and $3 \mathrm{D}$ computer graphics that give the user the experience of being present in a virtual environment" (International Bar Association, 2019).

Tomando en cuenta lo anterior, y siguiendo a Vera, Ortega y Burgos (2003), podemos definir la realidad virtual para efectos del presente artículo como "una simulación tridimensional dinámica en la que el usuario se siente introducido en un ambiente artificial que percibe como real en base a estímulos a los órganos sensoriales" (p. 4), agregando que dicha simulación tiene un origen informático y que emplea una amplia gama de herramientas de estimulación sensitiva.

Además, debemos tener en cuenta que la realidad virtual puede ser más o menos inmersiva, dependiendo del nivel de estimulación sensorial que genera sobre el individuo que interactúa con ella. En este sentido, se identifican a lo menos tres tipos de realidad virtual: de escritorio o no inmersiva, semi inmersiva y de inmersión total (Ai-Lim \& Wai, 2008). El primer tipo permite al individuo mantener una percepción distanciada de la simulación informática. El segundo, por su parte, provoca una inmersión ligera o moderada que fortalece la percepción del individuo. El tercero, finalmente, implica una inmersión de aspiración total que apunta hacia el pleno aislamiento de la persona del mundo exterior, centrando todas o la gran mayoría de sus capacidades sensoriales en la simulación respectiva (Ávalos, Camarena, \& Flores, 2014).

De este modo, la realidad virtual apunta a la generación de una realidad diversa que supone cierta autonomía de la realidad física en que vivimos. Esto es fundamental para entender la diferencia entre realidad virtual y realidad aumentada ya que, como bien señala la IBA, " $A$ R [augmented reality], on the other hand, creates a composite view by superimposing a computer-generated graphic onto the user's view of the real world" (International Bar Association, 2019).

La realidad aumentada, por tanto, se diferencia de la realidad virtual en razón de su grado de autonomía con respecto a la realidad física en que vivimos. Mientras la primera tiende a desprenderse de la realidad, la segunda pretende facilitar, mejorar y potenciar la percepción que tenemos de una realidad ya conocida. Así, siguiendo la definición de Wassom sobre realidad aumentada, "AR [augmented reality], then, is a blend of VR [virtual reality] with plain old physical reality. (...) It uses digital information to make our experience of actual, physical reality "greater". It doesn't create a brand new, standalone plane of 
Ricardo Vergara Olmos

Uso de Realidad Virtual y Realidad Aumentada en el Arbitraje Internacional

existence; it simply adds to the information we already process in the physical world" (Wassom, 2015). Este aspecto resultará de primordial importancia para nuestro análisis, como tendremos oportunidad de ver más adelante.

Ahora bien, en cuanto a los beneficios, si tomamos en cuenta la necesidad del árbitro de conocer y comprender los hechos del caso para aplicar el derecho, la utilidad de este tipo de tecnologías es evidente. No solamente resulta posible mejorar la capacidad de entendimiento del tribunal por medio de herramientas audiovisuales a través de la realidad aumentada, sino que además podemos lograr hacer "ver" al árbitro una situación que no pudo presenciar directamente, por medio de la realidad virtual. Ello se logra, desde luego, con la simulación que ofrece esta última tecnología, la cual permite reproducir de forma similar la serie de hechos que resultan relevantes para fallar la controversia sometida a arbitraje.

En otras palabras, ya no se le pide al juez que infiera a partir de evidencia indirecta o antecedentes que dan fe de la ocurrencia de un hecho, como podría ser una escritura pública o un video, sino que se enfrenta, de manera ficticia pero efectiva, a aquella porción de mundo que tomó lugar en un tiempo determinado y que no pudo percibir por los sentidos en aquel entonces, por no haberse encontrado en el lugar de la ocurrencia de los hechos.

De este modo, la realidad virtual y la realidad aumentada logran consagrarse como uno de los mayores avances para la administración de la justicia arbitral del último tiempo, en tanto son herramientas del tribunal para el conocimiento y mejor entendimiento de los hechos por una vía didáctica, y muchas veces, más clara.

\section{¿Por qué analizar estas herramientas tecnológicas en el marco del arbitraje internacional?}

El uso de realidad virtual y aumentada alcanza a toda la litigación, por constituir tecnología de aplicación general. Sin embargo, el análisis de su impacto en el arbitraje internacional resulta de particular interés, debido a las especiales características de los ámbitos en que se emplea este mecanismo de resolución de conflictos.

En primer lugar, es sabido que el arbitraje se reconoce y muchas veces se prefiere por el carácter experto de quienes actúan como árbitros (Born, 2016). Esto no solo apela a la pericia comercial por la que se caracterizan estos tribunales, sino además porque suelen responder efectivamente ante las características técnicas de las disputas que suelen ser sometidas a arbitraje. Así ocurre con el arbitraje de nombres de dominio o el arbitraje en materias deportivas, espacios que se manifiestan como "islotes de justicia" especializada (González de Cossío, 2014).

En segundo lugar, debemos tener en consideración quiénes suelen emplear este mecanismo de solución de controversias. Los litigantes dentro del arbitraje internacional tienden a ser personas, empresas o Estados que gozan de un gran patrimonio. Esto afecta directamente en el presupuesto de los litigantes para efectos del juicio arbitral, cuestión que impacta en la inversión empleada tanto en los medios probatorios, como en los mecanismos de persuasión utilizados durante el arbitraje para lograr un laudo favorable. 
En otras palabras, una capacidad económica superior permite al litigante acceder a ciertas herramientas de litigación más costosas, tales como los peritajes, los sistemas automáticos de organización y ordenación de documentos o, como en este caso, la tecnología de realidad virtual y aumentada, que sirve esencialmente para mejorar la capacidad de persuadir al tribunal arbitral respectivo y para ayudar en la probanza de los hechos.

Por último, debe tomarse en cuenta la promoción del empleo de estas tecnologías que ha efectuado la IBA tanto en su página web como en los distintos coloquios sobre arbitraje del último tiempo, muchos de ellos organizados por la International Chamber of Commerce (ICC), en los cuales se ha pretendido poner sobre la mesa el tema del uso de estos medios en el arbitraje (International Bar Association, 2019).

Sin duda, es un tema particularmente en boga dentro de la comunidad arbitral pero que, a pesar de ello, aún se encuentra en un estado incipiente de exploración. Por ello consideramos pertinente y necesario reflexionar sobre las cuestiones problemáticas del uso de realidad virtual y aumentada en el arbitraje, con el fin de proveer criterios de resguardo para la actividad del tribunal arbitral y delinear pautas de acercamiento con respecto a las tecnologías que puedan utilizarse a futuro en la litigación para lograr un efecto mayor de persuasión sobre los juzgadores.

\section{Cuestiones problemáticas del uso de realidad virtual y aumentada en el arbitraje internacional}

A pesar de la utilidad de la tecnología tanto para los litigantes en su tarea de persuadir, como para los árbitros en su tarea de juzgar, su empleo entraña más de un aspecto problemático que debe ser reflexionado y revisado con el fin de promover su uso adecuado en el proceso arbitral.

En esta sección plantearemos tres cuestiones problemáticas con respecto al uso de realidad virtual y aumentada en el arbitraje. En primer lugar, nos referiremos al efecto inmersivo de la realidad virtual, que afecta el modo por el cual el árbitro se relaciona con los acontecimientos relevantes de la controversia.

En segundo lugar, trataremos el problema del diseño de la simulación virtual, cuestión que tiene un efecto de reemplazo parcial sobre el árbitro como juzgador, a partir de la selección de la información relevante que constituye la base de la inferencia tecnológica desarrollada en aras de la producción de la simulación. Por último, en tercer lugar, nos referiremos a los problemas de costos que puede traer el empleo de estas tecnologías en el arbitraje internacional y su relación con los principios que informan el proceso arbitral.

\section{El efecto inmersivo de la realidad virtual: el árbitro como testigo}

Como señalamos en la segunda sección de este artículo, uno de los aspectos fundamentales de la realidad virtual es su efecto inmersivo para el usuario. Como señalan Gilson y Glennerster, "the ultimate goal of virtual reality is to present to the user computer-generated scenes in such a manner that the user should not be aware that they are in a virtual reality system at all” (Gilson \& Glennerster, 2012).

Es decir, la realidad virtual tiende a abandonar nuestra realidad para abarcar un entorno completamente independiente de ella. La efectividad de dicho objetivo puede ser medida a través de la noción de presencia, la cual corresponde a un concepto subjetivo relacionado con la psicología del usuario (Gutiérrez, Vexo, \& Thalmann, 2008). Gutiérrez, Vexo y Thalmann definen este concepto, señalando que "presence is 
Ricardo Vergara Olmos

Uso de Realidad Virtual y Realidad Aumentada en el Arbitraje Internacional

when the multimodal simulations (images, sound, haptic feedback, etc.) are processed by the brain and understood as a coherent environment in which we can perform some activities and interact" (p. 3).

Si bien esta noción supone el estar consciente sobre el carácter ficticio de la simulación, los mismos autores reconocen que la presencia puede llevar al envolvimiento y a reacciones emocionales por parte del usuario, afectando por tanto su percepción de la realidad que verdaderamente habita (Gutiérrez, Vexo, \& Thalmann, 2008). Es decir, la misma finalidad de la realidad virtual considera incidir en el usuario de modo que no solo se le otorga información, sino que también se moldea parte de su autopercepción a través de la generación de una experiencia completa que viene a adherirse a su propia identidad como persona.

Lo anterior no es menor si nos ubicamos en el contexto del arbitraje como método de solución de disputas que incluye la presencia de un tercero imparcial. Precisamente, un abandono de la distancia que caracteriza al juzgador de los hechos que suscitan la controversia, que viene acompañado además de elementos emocionales y, por tanto, personales, lo comprometen tanto en su imparcialidad como en su capacidad de apreciación crítica de los hechos.

Por eso es que el uso de realidad virtual resulta tan persuasivo al momento de presentar un caso ante un tribunal: logramos hacer que el juzgador se conecte de tal forma con el relato que le queremos contar, que lo hacemos parte de este. Dicho de otra manera, el árbitro deja de ser un juzgador para transformarse en algo así como un testigo. Curiosamente, una especie de testigo ex post facto. Y por tanto, comienza a cargar con la reacción emocional propia de quien observa directamente a pesar de no verse afectado por las consecuencias de la situación contemplada, como quien observa un robo o quien mira directamente un accidente automovilístico y siente temor o sorpresa.

No debemos olvidar graficar muy claramente el hecho que, como buen sucedáneo de testigo, al juzgador se le ofrece solo un punto de vista de los hechos, no los hechos en sí. Si bien en la argumentación jurídica y el relato de las partes ello también ocurre, dicha manifestación parcelada no toma lugar como lo hace una simulación virtual con aspiraciones de realidad. El efecto, desde luego, es mucho más fuerte con la tecnología analizada. De ahí nuestro particular interés en ella.

Es importante en este punto hacer presente la diferencia funcional entre el testigo y el juzgador en un proceso. Mientras el primero cumple un rol probatorio, facilitando el acceso del tribunal a la ocurrencia de los hechos a través de su testimonio (Bordalí, Cortez, \& Palomo, 2013). El segundo tiene la misión de ponderar e interpretar el relato emanado del testigo en tanto es potencial fuente de verificación y descubrimiento de los acontecimientos relevantes para la resolución de la controversia sometida a arbitraje, con el fin de alcanzar una decisión razonada sobre las solicitudes de las partes (Böckstiegel, 2003).

En definitiva, el cruce indebido y concentración en una misma persona de las funciones antes mencionadas obstaculiza una apreciación crítica de la prueba por parte del árbitro, generando una potencial prevalencia de la emocionalidad por sobre la argumentación al momento de la ponderación de la prueba de los hechos. 
Lo anterior no significa, desde luego, que el árbitro termine fallando contrario a derecho o que incurra en contradicciones al momento de efectuar su razonamiento jurídico. Ello sería excesivamente aventurado. Con todo, no podemos obviar el hecho de que esta forma de comprometer personal y psicológicamente al árbitro resulta riesgosa para un recto ejercicio de su rol como juzgador. Como mínimo, vemos una alteración en el ejercicio de dicha función.

De ahí que identifiquemos como primera cuestión problemática del empleo de realidad virtual en el arbitraje la desnaturalización del rol del árbitro en tanto juzgador, al convertirse en un testigo artificial ex post facto y al verse comprometido psicológicamente con los hechos de la disputa sometida a su arbitrio de una manera inapropiada en conformidad con sus fines en tanto tribunal.

\section{¿Quién pondera realmente la prueba? El reemplazo parcial del árbitro como juzgador}

El empleo de realidad virtual y aumentada supone la elaboración de un producto informático que, desde luego, no procede ni puede encontrar su origen en el tribunal mismo, cuyo rol es interpretar y ponderar la prueba, no producirla.

En consecuencia, el uso de esta tecnología significa dejar en las manos de las partes la fabricación de la simulación a ser utilizada en el arbitraje, partes que usualmente recurren a terceros expertos en el área debido al alto nivel de conocimiento y especialización que supone la generación de realidad virtual y aumentada.

Es en esta etapa de diseño y preparación del material explicativo y probatorio donde el árbitro puede verse indirectamente desplazado en cuanto a su calidad de juzgador, en la medida en que la realidad que muestra la simulación no es una interpretación del árbitro sino, más bien, del abogado de cada parte y su respectiva teoría del caso.

Cuando hablamos de teoría del caso, nos referimos a "la idea central que adoptamos para explicar y dar sentido a los hechos que se presentarán como fundantes de una historia, permitiendo dar cuenta de la existencia de una determinada teoría jurídica" (Blanco, Decap, Moreno, \& Rojas, 2005, p. 18). O bien, en términos más simples, hablamos del "relato simple, hilado, único, coherente y creíble de los hechos que van a ser materia del juicio" (Alcaíno \& Cortés, 2012, p. 47).

Así, como cada parte tendrá una teoría del caso propia, los abogados respectivos no pedirán simplemente que se simule una situación a secas, sino que (si son lo suficientemente astutos y diligentes) solicitarán expresamente la aplicación de su teoría del caso a la forma de representar la realidad que buscan mostrar al tribunal arbitral.

Por tanto, el hecho de que la simulación busque dar cuenta de una realidad hipotética que no arranca de cuestiones específicas como documentos o pruebas periciales sino solo en forma relativa, tiene por efecto que la teoría del caso de cada parte pueda potencialmente transformarse en una realidad autónoma y a veces excesivamente sugerente para con el tribunal.

Podría advertirse que, en cierto modo, la simulación no dista demasiado del testigo, en tanto tampoco le consta al tribunal arbitral la existencia real de aquello que el testigo relata como una ocurrencia auténtica 
Ricardo Vergara Olmos

Uso de Realidad Virtual y Realidad Aumentada en el Arbitraje Internacional

de los hechos. Sin embargo, debemos recordar que, a diferencia de la declaración de un testigo, la realidad virtual y hasta cierto punto también la aumentada tienen un efecto inmersivo que afecta de manera diversa la percepción del juzgador, ejerciendo una mayor influencia como medio de prueba, que incluso puede llevar a la confusión o a un compromiso emocional inapropiado para un tercero imparcial.

Pero además del desplazamiento interpretativo ocasionado por la forma en que el abogado solicita la aplicación de su teoría del caso en la simulación, debemos tomar en cuenta que el mismo diseñador de la simulación realiza a la vez una interpretación de la solicitud del abogado de parte que pide el producto. Por mucho que la defensa de una de las partes informe detalladamente al proveedor de la simulación sobre qué espera de él, el sentido común da cuenta de que la misma empresa encargada de fabricar esta realidad virtual o aumentada va a poner parte de sus ideas en la elaboración del producto, en la medida en que no se vea impedida por quien realiza el encargo.

Lo anterior deriva en que no solo hay una, sino dos interpretaciones previas a la formulación de la simulación que finalmente observará el tribunal arbitral y sobre la cual formará su opinión con respecto a los hechos sustanciales de la controversia.

Por lo demás, como advierte con lucidez parte de la doctrina, muchas veces los técnicos convocados para, en principio, ilustrar al tribunal arbitral sobre materias técnicas, suelen ponerse totalmente de parte de quien los contrata (González de Cossío, 2014). Ello no es raro, si tomamos en cuenta que finalmente están ofreciendo un servicio que solo interesa a la parte respectiva en la medida en que le sea favorable a su teoría del caso. Si, por ejemplo, en la actividad de peritos ello es manifiesto, en el caso de la realidad virtual y aumentada resulta evidente.

De este modo, el tribunal arbitral se aleja cada vez más de una lectura limpia y transparente de los hechos, ajena a interpretaciones antojadizas de las partes que influyen en la realidad finalmente mostrada a los juzgadores. Es por ello que identificamos como segunda cuestión problemática del uso de realidad virtual y aumentada en el arbitraje internacional el distanciamiento del tribunal de los hechos, lo que de otro modo puede entenderse como el reemplazo parcial del árbitro como juzgador.

\section{Los costos elevados y el trato igualitario de las partes}

Los costos del arbitraje siempre han sido un tópico de relevancia para los abogados practicantes en esta materia (Bantekas, 2015). Ello se debe a que, a diferencia de la justicia estatal que implica un servicio gratuito para la solución de conflictos, el arbitraje es un mecanismo alternativo de solución de disputas de carácter privado que contempla naturalmente la remuneración de los árbitros (Vásquez, 2018). A lo anterior se suman costos típicos de la litigación, como la producción de documentos y el uso de medios probatorios como los peritajes (Carey \& Fernández, 2014), y, en este caso, el uso de realidad virtual y aumentada como método de persuasión del tribunal arbitral. Desde esta perspectiva, resulta interesante observar qué ocurre con la igualdad de las partes con referencia a los costos del uso de realidad virtual y aumentada, en atención a que esta tecnología podría resultar más costosa que otros medios probatorios tradicionales.

La igualdad de las partes es un principio transversalmente protegido en los distintos ordenamientos jurídicos y en los instrumentos internacionales que regulan el arbitraje (Waincymer, 2012). Así, por 
ejemplo, podemos encontrarlo en el artículo 9.2 (g) de las IBA Rules on the Taking of Evidence in International Arbitration sobre admisibilidad de la prueba, al señalar que el tribunal arbitral podrá excluir evidencia en base a la protección de la igualdad de las partes. Lo mismo puede observarse en el artículo 18 de la Ley Modelo de la CNUDMI sobre Arbitraje Comercial Internacional, que señala que deberá tratarse a las partes con igualdad y darse a cada una de ellas plena oportunidad de hacer valer sus derechos. Por nuestra parte, consideramos poco probable que los costos del empleo de esta tecnología resulten problemáticos para los efectos de la igualdad de las partes en el arbitraje internacional. Ello se funda en que, como señalamos anteriormente, las partes de estos procesos suelen tener las facultades económicas suficientes como para invertir en este tipo de métodos de demostración y persuasión ante el tribunal arbitral. No obstante, el principio de igualdad procesal debe por supuesto seguir de pie y tomarse en cuenta en situaciones donde eventualmente pudiera existir un desbalance considerable entre las partes. Es decir, la garantía de este principio debe ponderarse acorde a las circunstancias.

En este punto, resulta importante tener presente que el empleo de esta tecnología tiene más bien el efecto de intensificar la propuesta argumentativa de las partes y sus medios de prueba, que probar por sí solo un punto. Lo que se logra es que, a través de la agudización de los sentidos y el compromiso perceptivo del juzgador, ya sea a través de una imagen superpuesta a una imagen real (como en la realidad aumentada) o una simulación completa de lo que probablemente ocurrió en base a las circunstancias conocidas, apoyando una inferencia para efectos de una presunción (como en la realidad virtual), se aumente el nivel de persuasión efectiva del discurso argumentativo presentado ante el tribunal.

Al ser una cuestión de grado, el análisis no puede sino ser casuístico, siendo tarea del tribunal arbitral determinar la eventual afectación del principio de igualdad. Ahora bien, el tribunal no tiene por qué saber que un gasto dentro del litigio puede resultar excesivo a una de las partes, por lo que, con base en una lógica dispositiva, debiera ser carga de las partes hacer presente al tribunal que carece de medios para igualar el método probatorio de la contraparte, o bien, objetarlo. Esto supone, a su vez, que la parte que se disponga a emplear un método expositivo que pudiera implicar excesiva falta de equivalencia de formato con respecto a la otra, tenga la obligación, con base y en resguardo del principio de igualdad, de informarlo así al tribunal y a la contraparte, para evitar un desequilibrio en la ritualidad argumentativa del juicio so pena de verse inhibido por el tribunal de emplear el formato no informado. Esta perspectiva, desde luego, debe ser tomada con cautela por ser un posible foco de alegaciones de indefensión o afectación indebida del derecho a la defensa del que se ve limitado en su exposición.

Prima facie esto podría sonar excesivamente paternalista e incluso limitativo de la libertad de defensa de las partes. Sin embargo, un par de ejemplos pueden ilustrar analógicamente la posible situación de inequidad de las partes, considerando que la realidad virtual y aumentada tienen un efecto psicológico superior que los medios de prueba tradicionales.

Como primer ejemplo, podemos considerar que ambas partes de un arbitraje han presentado escritos al tribunal haciendo presentes sus argumentos sobre la controversia. Sin embargo, solo una de ellas puede acudir a realizar sus alegatos en persona, mientras que la otra se encuentra imposibilitada debido a una medida sanitaria que le impide viajar a las oficinas del árbitro.

Cualquiera diría que, si es posible esperar a que ambas partes aleguen, debiera esperarse. Si no es así, algunos podrían considerar injusto permitir que solo una parte realice alegatos. ¿Por qué? Porque no es 
Ricardo Vergara Olmos

Uso de Realidad Virtual y Realidad Aumentada en el Arbitraje Internacional

lo mismo argumentar por escrito que oralmente. El efecto sobre el juzgador es distinto, y es una oportunidad explicativa y de surgimiento de preguntas del tribunal que resulta ser de suma importancia. Otro ejemplo es si ambas partes presentan escritos y acuden a realizar alegatos. Con anterioridad, se había acordado por las partes frente al árbitro que los alegatos no contarían con apoyo visual. Sin embargo, una de ellas acude con una presentación en Power Point con gráficos e imágenes sugestivas y el árbitro decide permitir la exposición.

En este caso claramente estamos ante una situación injusta: una parte incumple una promesa para tomar ventaja de otra. ¿Pero qué es lo que nos preocupa realmente? Ambas partes van a poder argumentar, de eso no cabe duda. Incluso verbalmente, respondiendo preguntas del árbitro. Pero una de ellas podrá interactuar injustamente mejor que la otra con el juzgador, porque no solo cuenta con el lenguaje verbal y paraverbal del alegato oral, sino que además posee material visual que mejora su explicación y al que no ha podido acceder la otra parte.

Como tercer ejemplo, ambas partes presentan escritos, acuden a realizar alegatos y presentan el mismo video de un accidente que causó una explosión en una fábrica para hacer comentarios. Sin embargo, una de ellas ha editado el video utilizando técnicas de realidad aumentada, por lo que cada cosa que explica tiene un reflejo directo en el video.

En rigor, es la misma evidencia. Sin embargo, el retoque genera una correlación entre la argumentación y la imagen que genera efectos ajenos a la mera discusión de un video sin adiciones. Al igual que en los ejemplos anteriores, existe una falta de equivalencia de formato de interacción con el tribunal, pero aquí se genera una imagen que aparentemente reacciona al discurso argumentativo de una de las partes. $\mathrm{Al}$ incluir elementos de movilidad y probablemente de sonido, influye emocionalmente más que entregar información nueva al juzgador (Armstrong, 2010), alterando su comprensión de un mismo suceso solamente por el aparente manejo de los efectos percibidos en el material audiovisual.

Como cuarto ejemplo, podemos considerar lo mismo que el tercer ejemplo, pero ahora una de las partes agrega una simulación completa de lo que, hipotéticamente, habría ocurrido con posterioridad o anterioridad al accidente, en base a lo que se muestra en el video, mientras que la otra no ha podido acceder a dicha técnica de exposición.

Aquí es donde probablemente la falta de equilibrio en la defensa se hace más notoria: una parte muestra algo que la otra solo puede verbalizar, y además, todo lo que relate el que expone con realidad virtual ocurre precisamente de ese modo en la simulación presentada. Este efecto puede contenerse o morigerarse para el juzgador si, como contrapartida, le presentan otra versión plasmada en una simulación que pudiera potencialmente afectarle del mismo modo en los sentidos. Sin embargo, en caso de que una de las partes no pueda acceder a dicha tecnología, la balanza se verá injustamente desequilibrada precisamente en virtud de un problema de costos de implementación de tecnología.

Con los ejemplos anteriores no pretendemos decir que cada vez que alguien desee exponer con técnicas novedosas o que impliquen un costo superior a lo normal tenga que verse limitado o privado en el ejercicio de su defensa. El respeto al principio de igualdad de las partes dentro del proceso arbitral no supone necesariamente igualar la cantidad de recursos que cada parte pueda o quiera destinar al empleo de tecnología en el ámbito probatorio. Sin embargo, el respeto del principio de igualdad de las partes sí 
supone, siguiendo un criterio prospectivo frente a las tecnologías que aumentan la sensibilidad del juzgador, que se tenga presente desde un principio el potencial uso de dichas tecnologías en el proceso para equiparar los canales comunicativos a emplear por las partes, es decir, los medios por los cuales van a entregar su mensaje.

Si no pudiera equipararse la capacidad comunicativa de las partes, y ello trajera por consecuencia un desmedro significativo en la igualdad de armas de las partes, debiera excluirse la tecnología avanzada como apoyo para la demostración o presentación de evidencia en juicio. En otras palabras, el principio de igualdad de las partes prevalecería frente al derecho a la libertad de exposición de la defensa, en aras del mantenimiento de un proceso justo y en equilibrio. Al fin y al cabo, como mecanismo de intensificación de la argumentación y los medios de prueba, el empleo de esta tecnología supone partir de alguna base a lo menos indiciaria para poder producir la simulación o aumento de realidad. No es indispensable, pero sí puede resultar de gran ayuda y hacer una importante diferencia.

Sin perjuicio de lo anteriormente expuesto, y debido a las pocas dificultades que parece presentar en la práctica, consideramos que este resulta ser más bien un punto que excepcionalmente requerirá atención, sin constituir de por sí uno de los mayores problemas del empleo de realidad virtual y aumentada en el arbitraje internacional.

\section{Algunas observaciones y recomendaciones para un uso adecuado de la realidad virtual y la realidad aumentada en el arbitraje internacional}

Toda nueva tecnología supone un desafío, y como tal, merece ser superado, no abandonado por la dificultad o las incomodidades que pueda generar en el medio en el cual dicha tecnología se desarrolla.

De ahí que, en nuestra opinión, la manera en que debe enfrentarse el uso de realidad virtual y realidad aumentada no debe apuntar hacia la prohibición o la obstaculización innecesaria de su implementación sino, por el contrario, hacia su utilización responsable, consciente y precavida. Al fin y al cabo, permite a las partes hacer una exposición más persuasiva de sus respectivas posturas, y faculta al tribunal para tener un acercamiento más didáctico y reflexivo sobre la evidencia del caso concreto.

A continuación se exponen algunas observaciones y recomendaciones pertinentes para el uso adecuado de la realidad virtual y la realidad aumentada en el arbitraje internacional. Se explicará la necesidad del análisis de las fuentes y la metodología de producción de la simulación respectiva, se argumentará en favor de la preferencia del uso de realidad aumentada por sobre la realidad virtual, se propondrá una aproximación hacia estas técnicas como mecanismos de comprobación consecuentes, se invitará al lector a tomar una postura especialmente cautelosa y a la vez activa ante las nuevas tecnologías en la litigación y se ahondará brevemente en las posibles consecuencias del incumplimiento de los criterios y recomendaciones propuestas.

\section{Necesidad de análisis de fuentes y metodología de producción de la simulación}

Un primer paso para evitar un sesgo epistemológico frente a los verdaderos hechos del caso, debido a la utilización de simulaciones, es el análisis de las fuentes empleadas en la elaboración de estas y el conocimiento de su metodología de producción. 
Ricardo Vergara Olmos

Uso de Realidad Virtual y Realidad Aumentada en el Arbitraje Internacional

En este sentido, resultan útiles los criterios planteados por Susan Haack a propósito del empleo de evidencia epidemiológica en el ámbito de los daños tóxicos o toxic torts. Lo anterior, puesto que a través de estos criterios también se busca evitar un sesgo cognitivo de parte del juzgador al enfrentarse a un producto técnico complejo, como es el análisis estadístico epidemiológico, para cuyo arribo se deben tomar una multiplicidad de factores que no necesariamente se conocen en principio por el tribunal.

Dichos criterios son tres: fiabilidad, relevancia y exhaustividad (Haack, 2018). A través de ellos, se busca determinar que el análisis estadístico esté realizado sobre una base científica sólida y sin sesgos cognitivos relevantes. Si extrapolamos dichos criterios al caso de la realidad virtual y la realidad aumentada, podemos obtener las siguientes conclusiones.

En cuanto a la fiabilidad, resulta útil a los árbitros saber de dónde las partes han obtenido la información a partir de la cual recrean la escena que luego muestran por medio de la simulación al tribunal arbitral. ¿Se basan solamente en la información de testigos? ¿Han empleado videos que también pueda ver el tribunal? ¿Han efectuado cálculos matemáticos que permitan aseverar que de ese modo y solo de ese modo podían desarrollarse los hechos aludidos? ¿Existen documentos que den cuenta de la forma en que se muestran los hechos en la simulación? ¿Existe información confidencial sobre la cual se basa el producto informático?

Todos estos antecedentes, en nuestra opinión, deben estar disponibles para el tribunal con el fin de justificar la simulación, siempre que la misma pretenda emplearse con un efecto probatorio y no meramente persuasivo. Dichos antecedentes constituyen información que el tribunal arbitral debe estar facultado para solicitar con el fin de proteger su generación de convicción sobre la prueba rendida por las partes.

En cuanto a la relevancia, para los efectos de la aplicación del criterio al arbitraje, debe entenderse tanto en su arista procesal de sustancialidad para la resolución del conflicto como de finalidad del empleo de la tecnología. De modo que, si una parte busca probar hechos que no son relevantes para juzgar la controversia, no hay duda de que la prueba respectiva debe ser excluida. Pero a su vez, si el empleo de la tecnología respecto a un punto de prueba solo busca comprometer emocionalmente a los árbitros más que explicarles hechos complejos cuya demostración o explicación se facilita por la tecnología, también estaremos ante un empleo irrelevante de la misma. Tomando como base que la introducción de tecnología debe ser una facilitación y mejoramiento de la tarea del juzgador por sobre un método de alteración de su criterio, la relevancia protege la integridad emocional que debe cargar el carácter imparcial del juzgador. Así, si el tribunal arbitral mantiene presentes los puntos de prueba y su funcionalidad con respecto al laudo, resulta posible evitar prestar excesiva atención a puntos que no aportan a la decisión de los árbitros y prevenir un excesivo compromiso psicológico e identitario con los hechos que se presentan en razón de la controversia.

Por último, en cuanto a la exhaustividad, si la aplicamos al caso de la realidad virtual y la realidad aumentada, debemos recordar que ambas tecnologías suponen un alejamiento de la realidad: la primera de forma completa, la segunda de forma relativa. Con la exhaustividad como criterio de protección de la calidad de juzgador de los árbitros, debemos tener siempre presente que en su calidad de simulaciones, los productos de realidad virtual y realidad aumentada no comprenden todos los hechos relevantes para la decisión de la controversia. 
De ahí que, con justo motivo, el tribunal deba realizar las preguntas de rigor a las partes que presenten un material de este tipo para verificar si falta el tratamiento de ciertos aspectos fácticos que, existiendo indicios de haber concurrido, no fueron reproducidos en la simulación respectiva. Por ejemplo, quizá sería positivo también, para evitar sesgos, que se reprodujeran hechos ocurridos antes o después de aquel presentado en la ficción tecnológica. Si nos preocupamos de velar por la bilateralidad de la audiencia, también será necesario dotar a la contraparte de una oportunidad precisa para hacer ver estos aspectos de eventual insuficiencia de la simulación presentada por la contraparte. Esto puede calzar sin problema alguno, en nuestra opinión, en las observaciones a la prueba.

Solo así pueden evitarse sesgos que, si bien también pueden generarse con otros medios de prueba como los testigos, donde se combate el problema a través de las repreguntas o la contra examinación, en este caso se presentan con pretensiones de realidad superiores que generan un efecto psicológico mayor en los juzgadores, lo que justifica el resguardo.

Atendiendo a los criterios antes expuestos de fiabilidad, relevancia y exhaustividad, el tribunal arbitral podrá proteger su labor como juzgador a partir de un análisis riguroso del material audiovisual simulado que se le presente, evitando caer en sesgos cognitivos o epistemológicos que lo priven de tener claridad sobre los hechos de la causa.

\section{Preferencia de la realidad aumentada por sobre la realidad virtual}

Difícilmente puede negarse la capacidad de la realidad virtual de mostrarnos lo que no pudo ser observado, del efecto de testigo expost facto y de inmersión y presencia antes señalados. Sin embargo, son estas mismas características las que hacen peligrar mayormente la labor del tribunal arbitral al momento de juzgar los hechos materia del caso cuando se utiliza realidad virtual.

Ello no ocurre directamente con la realidad aumentada. Como señalamos anteriormente, la realidad aumentada no se desprende por completo del mundo que habitamos, sino que logra mejorar la capacidad de percepción y comprensión del individuo que interactúa con ella respecto a una situación determinada, a través de medios audiovisuales.

Desde luego, esa forma de acercamiento o efecto explicativo no siempre estará limpia de sesgos o sugerencias. A veces, incluso si se está "aumentando la realidad", puede que el modo en que se haga o se presente al juzgador lo lleve a tomar una decisión muy distinta a la que tendría con otra perspectiva de aumento de realidad.

Sin perjuicio de ello, el hecho de que el tribunal pueda mantenerse cerca de la realidad efectiva y no esté observando una simulación que pretende ser completamente autónoma de los hechos verdaderos, permite que la labor de juzgador de los árbitros se vea mayormente protegida con el uso de realidad aumentada por sobre el uso de realidad virtual.

Para graficar de mejor manera este punto, resulta útil hacer una comparación. No es lo mismo una simulación vía realidad virtual que emule el choque de dos automóviles, permitiendo acercarnos a los detalles de la colisión, el modo en que se destruyen los vehículos o los efectos del impacto en los pasajeros, que una simulación efectuada sobre el video mismo en que consta el choque de los dos automóviles 
Ricardo Vergara Olmos

Uso de Realidad Virtual y Realidad Aumentada en el Arbitraje Internacional

donde, sobre las mismas imágenes reales, se plasmen figuras audiovisuales que faciliten la percepción y entendimiento del tribunal sobre los hechos.

Por supuesto, lo anterior supone que exista otro medio de prueba, principalmente videos o imágenes que operen como base para la superposición de herramientas audiovisuales como las antes descritas. A falta de dicho material, la realidad virtual sigue siendo una opción, pero más riesgosa para efectos de los sesgos cognitivos y epistemológicos del tribunal.

De este modo, es recomendable dar un carácter residual a la realidad virtual, o emplearla al menos solo con fines didácticos por sobre la idea de hacer de ella un medio de prueba propiamente tal, siendo preferible el empleo de realidad aumentada por tener un carácter más conservador y cercano a la realidad fáctica y no simulada.

\section{La realidad virtual y la realidad aumentada como mecanismos de comprobación consecuente}

Todo medio de prueba auténtico, en cuanto evidencia efectiva de la ocurrencia de un hecho, supone un sustrato fáctico a partir del cual se conforma y un soporte que permite la revisión posterior de aquello cuya existencia sustenta con su relato. Así, por ejemplo, cuando hablamos de la prueba pericial, el perito se pronuncia sobre una serie de hechos o circunstancias a partir de los cuales emana el análisis técnico contenido en su informe; y cuando hablamos de la prueba testimonial, el testigo se refiere a los hechos que ha percibido por sus sentidos y que constituyen su testimonio.

¿Pero qué ocurre con la realidad virtual y la realidad aumentada? ¿De dónde obtienen el sustrato fáctico necesario sobre el cual desenvuelven sus cualidades demostrativas?

Se debe distinguir, por una parte, que la realidad aumentada supone una base fáctica visual, auditiva o sensorial en general sobre la cual desarrollarse, y necesita una realidad que aumentar. En consecuencia, su ámbito de aplicación va a recaer sobre otros medios de prueba, y por tanto, se va a manifestar especialmente como un mecanismo de comprobación consecuente y agudizada de otro medio ya disponible para los litigantes. Así, por ejemplo, ocurrirá cuando se detallen por esta tecnología las imágenes de un video con acercamientos o gráficos superpuestos, o cuando se enfaticen ciertos ruidos frente a otros en un audio, con el fin de evidenciar a partir de prueba documental ciertas cuestiones que podrían pasar desapercibidas sin este apoyo tecnológico para el juzgador.

Por otra parte, la realidad virtual también va a necesitar un sustrato fáctico, pero a partir de él, va para comenzar autónomamente un desenvolvimiento demostrativo de la teoría del caso del litigante respectivo. Debemos recordar que, a diferencia de la realidad aumentada, la realidad virtual busca generar una realidad diversa con vocación de autonomía de la realidad física a la que pertenecemos. De por sí, la realidad virtual supone un mecanismo contra fáctico que puede o no tener correlato en la realidad.

Vista como un mecanismo de comprobación consecuente, aparece como complemento de un medio probatorio altamente empleado ante la falta de hechos completos: las presunciones. En efecto, las presunciones corresponden a procedimientos lógicos empleados por el juez, que parten de un hecho conocido llamado base, para alcanzar el conocimiento o inferir la ocurrencia de hechos desconocidos (Casarino, 2005). La realidad virtual es un excelente complemento consecuencial a este tipo de medio de 
prueba. A la vez, al emplearse como expresión hipotética de la parte de un silogismo y no como una reformulación de evidencia directa (fotografías o videos de un hecho) o formulación sin correlato fáctico alguno, deviene en una utilización más segura para efecto de la integridad cognitiva del juzgador.

Como es posible apreciar, cada tecnología se asocia a otros medios de prueba. Mientras la realidad aumentada puede complementar a la prueba documental y pericial, la realidad virtual permite graficar con mayor precisión las inferencias lógicas a las que se invita al tribunal arbitral cuando, a través de la argumentación de parte, realiza presunciones. Esta asociación permite la seguridad de partir de una base menos riesgosa en cuanto a la influencia emocional que pueda generar en los árbitros, y se complementa con los criterios ofrecidos en el primer subtitulo de esta misma sección.

Dicho de otro modo, concebir a la realidad virtual y la realidad aumentada como mecanismos de comprobación consecuente a otros medios de prueba, es una aproximación que propende a la seguridad en el uso de dichas tecnologías y a la utilidad de su empleo en el arbitraje.

\section{Especial cautela y postura activa frente a las nuevas tecnologías en la litigación}

Aunque resulta evidente la necesidad de tener cautela frente a las nuevas tecnologías, resulta positivo encauzarla hacia un sentido comunitario activo dentro de la jurisprudencia arbitral internacional. Con esto nos referimos a que es particularmente recomendable a los árbitros el estar al tanto de la experiencia que sus otros colegas han tenido en el empleo de esta tecnología, así como compartir sus respectivas reflexiones y encuentros o desencuentros con estos métodos de manejo de la evidencia en el litigio arbitral.

Esto se logra, desde luego, con una actitud activa dentro de la comunidad arbitral internacional, participando de los distintos foros de discusión respectivos e informándose del modo en que opera la nueva tecnología. Los árbitros deben tomar ventaja de la promoción e información que entregan organismos tales como la ICC o la IBA al respecto, atendido su deber de administración de justicia a través del arbitraje.

Por lo demás, tener una formación constante en nuevas tecnologías resulta particularmente atractivo en el ámbito del arbitraje, cuyo carácter personalizado y ergonómico frente al interés de los litigantes favorece el uso de procedimientos y métodos menos limitados que aquellos que imponen ciertos ordenamientos domésticos.

Así, un criterio atento y una postura activa frente a las nuevas tecnologías en la litigación favorecerán a la larga la introducción de estos medios al proceso.

\section{Consecuencias de seguir o no los criterios y recomendaciones propuestas}

Los criterios y recomendaciones propuestos permiten en su conjunto no solo orientar el empleo de la tecnología en comento, sino también la actitud de los árbitros frente a la misma en las audiencias que les toque dirigir y el ejercicio de los derechos que corresponda desempeñar a los abogados de parte. 
Ricardo Vergara Olmos

Uso de Realidad Virtual y Realidad Aumentada en el Arbitraje Internacional

Pero además, permiten hacer frente principalmente a los problemas identificados, esto es, el árbitro como testigo y el desplazamiento del árbitro como juzgador. En cuanto a los costos y el trato igualitario, como ya señalamos, no parece ser un problema que tendrá mucha presencia en la práctica, y que en cualquier caso, deberá resolverse conforme a la desigualdad relativa presente en cada caso.

Los criterios de fiabilidad, relevancia y exhaustividad sobre análisis de las fuentes y la metodología de producción de la simulación respectiva, facultan al árbitro para evitar ser desplazado en su tarea de juzgar al identificar los puntos en que las partes o los diseñadores han tomado decisiones que pudieran afectar los hechos que se busca recrear. Conocer los materiales de construcción permite entender cómo se han manipulado los elementos entorno a la estructura. Alejarse de dichos criterios podría llevar a que los árbitros terminen juzgando con base en simulaciones virtuales que no se condicen real o suficientemente con lo que en los hechos pasó.

En cuanto a preferir el uso de realidad aumentada por sobre la realidad virtual, debemos concebirlo como una manera de disminuir el riesgo de exposición a un alejamiento excesivo de otros medios de prueba más directos. No seguir este criterio puede traer consecuencias más o menos relevantes dependiendo del contexto completo del asunto en cuestión. En cualquier caso, es más bien un criterio preventivo, para sostener el juicio sobre bases más concretas.

Respecto del criterio referido al entendimiento de la realidad virtual y la realidad aumentada como mecanismos de comprobación consecuente, se debe advertir que no tenerlo presente puede traer por efecto que en el ejercicio de su facultad de apreciar la prueba, el árbitro confunda la mera afirmación con la prueba de los hechos. Ligar la realidad virtual y aumentada con un medio de prueba de carácter más directo permite, en cierto modo, aferrarse a la realidad y sostenerse de antecedentes que ejercen una influencia menos riesgosa sobre el juzgador. Así, la tecnología se vuelve más una herramienta que un artificio para seducir el razonamiento del árbitro. Por ello, es plenamente recomendable entender esta tecnología como un mecanismo de comprobación consecuente.

Por último, la invitación al lector a tener una especial cautela y una postura activa en el conocimiento de las nuevas tecnologías es una recomendación que, de no ser tomada, no necesariamente va a traer efectos negativos. Pero sí va a demorar el avance en el uso adecuado y seguro de estos medios. Es por ello que resultaría positivo obtener no solo en esta área, sino en general, una actitud de colaboración dentro de la comunidad arbitral para propender el mejoramiento de los mecanismos de solución de disputas de que tomamos parte, como efectivamente han invitado a hacer las organizaciones gremiales de arbitraje en distintos países del mundo.

\section{Conclusiones}

A diferencia de otros métodos de presentación de la prueba, la realidad virtual y la realidad aumentada muestran un mayor potencial de generación de sesgos cognitivos y epistemológicos sobre los juzgadores. Sin embargo, estamos ante una oportunidad, no ante un problema. El uso de realidad virtual y realidad aumentada debe ser bienvenido en el proceso arbitral internacional, porque permite al tribunal clarificar los hechos sobre los cuales deberá resolver la controversia sometida a su conocimiento con una mayor calidad explicativa. Con su uso, las partes amplían el abanico de posibilidades argumentativas y 
demostrativas, e incluso, vuelven aún más palpable la evidencia con la que cuentan por medio de una reformulación sensitiva que, además, apoya la labor del juzgador.

Sin embargo, si por algo se caracteriza un buen jurista es por su prudencia. En consecuencia, el empleo de estas nuevas tecnologías debe realizarse con mesura, rigurosidad y cuidado, tanto en el ámbito probatorio como en el espacio del foro reservado para la persuasión de los árbitros. De ahí que resulte pertinente y útil tomar en cuenta las recomendaciones antes expuestas con el fin de lograr el objetivo de una aplicación efectiva y fructífera de las tecnologías mencionadas, en el medio arbitral.

Finalmente, cuando analizamos la prueba, no solo debemos observar el cariz principal de lo que se nos muestra, sino que debemos aprehender el método por el cual se nos hace llegar la información, el por qué se nos muestra de ese modo y qué tan completa resulta la exposición para efectos de fallar la controversia. Solo teniendo claridad respecto de ello podremos mantener intacta la correcta función compositiva del proceso arbitral, y también, aprovechar las bondades que la tecnología nos ofrece al momento de resolver una controversia para obtener el mejor y más justo de los resultados para las partes.

\section{Referencias}

Ai-Lim, E., \& Wai, K. (2008). A Review of Using Virtual Reality for Learning. In A. El Rhabili, Transactions on edutainment I (pp. 231-241). Berlin: Springer.

Alcaíno, R., \& Cortés, M. (2012). Curso Elemental de Litigación Oral. Santiago: Editorial Jurídica de Chile.

Armstrong, S. (2010). Persuasive Advertising. Evidence-based principles. Chippenham: PalgraveMacMillan.

Ávalos, E., Camarena, P., \& Flores, J. (2014). La realidad virtual, una tecnología innovadora aplicable al proceso de enseñanza de los estudiantes de ingeniería. Apertura, 6(2). Retrieved from http://www.udgvirtual.udg.mx/apertura/index.php/apertura/article/view/547

Bantekas, I. (2015). An Introduction to International Arbitration. Cambridge: Cambridge University Press.

Blanco, R., Decap, M., Moreno, L., \& Rojas, H. (2005). Litigación Estratégica en el Nuevo Proceso Penal. Santiago: Lexis Nexis.

Böckstiegel, K.-H. (2003). The Role of the Arbitrators in Investment Treaty Arbitration. In A. J. Van den Berg, International Commercial Arbitration: Important Contemporary Questions. La Haya: Kluwer Law International.

Bordalí, A., Cortez, G., \& Palomo, D. (2013). Proceso civil. El juicio ordinario de mayor cuantía. Santiago: Thomson Reuters.

Born, G. (2016). International Arbitration: Law and Practice ( $2^{\circ}$ ed.). Londres: Wolters Kluwer.

Brudniy, A., \& Demilhanova, A. (2012). The Vritual Reality in a Context of the "Mirror Stage". International Journal of Advances in Psychology, 1, 6-9. Retrieved from http://www.ijpsychol.org/ 
Ricardo Vergara Olmos

Uso de Realidad Virtual y Realidad Aumentada en el Arbitraje Internacional

Burgos, M. Á., Ortega, J. A., \& Vera, G. (2003). La realidad virtual y sus posibilidades didácticas. Etic@net, 1-17.

Carey, J., \& Fernández, G. (2014). Los costos en el arbitraje. In E. Picand, Estudios de arbitraje en homenaje a Patricio Aylwin Azócar (pp. 163-194). Santiago: Thomson Reuters.

Casarino, M. (2005). Manual de derecho procesal (Derecho Procesal Civil) (6º ed., Vol. III). Santiago: Editorial Jurídica de Chile.

Gilson, S., \& Glennerster, A. (2012). High Fidelity Immersive Virtual Reality. In X.-X. Tang, Virtual Reality - Human Computer Interaction (pp. 41-58). Rijeka: InTech.

González de Cossío, F. (2014, Abril 28). Arbitraje deportivo en iberoamérica. Retrieved from González de Cossío Abogados: Publicaciones: http://www.gdca.com.mx/espanol/publicaciones/arbitraje.html

González de Cossío, F. (2014). El prurito del perito. Ius et praxis(45), 207-215.

Gutiérrez, M., Vexo, F., \& Thalmann, D. (2008). Stepping into Virtual Reality. Londres: Springer.

Haack, S. (2018). In the Epistemological Spotlight: Epidemiological Evidence in Toxic Torts. In I. C. Procesal, XV Congreso Panameño de Derecho Procesal (pp. 361-378). Chitré: Instituto Colombo Panameño de Derecho Procesal.

International Bar Association. (2019, Septiembre 15). Technology Resources for Arbitration Practitioners. Retrieved from https://www.ibanet.org/technology-resources-for-arbitration-vrar.aspx

Vásquez, M. F. (2018). Tratado de arbitraje en Chile. Arbitraje interno e internacional. Santiago: Thomson Reuters.

Wahab, M., \& Katsh, E. (2018). Revolutionizing Technologies and the Use of Technology in International Arbitration: Innovation, Legitimacy, Prospects and Challenges. In M. Piers, \& C. Aschauer, Arbitration in the Digital Age. The Brave New World of Arbitration (pp. 27-55). Cambridge: Cambridge University Press.

Waincymer, J. (2012). Procedure and Evidence in International Arbitration. Alphen aan den Rijn: Wolters Kluwer.

Wassom, B. (2015). Augmented Reality Law, Privacy and Ethics. Law, Society, and Emerging AR Technologies. Waltham: Elsevier.

(c) (1) ()

Open Access This article is licensed under a Creative Commons Attribution-Non Commercial 4.0 International License, which permits the use, adaption and sharing as long as you give appropriate credit to the original author(s) and the source. The images or other third party material in this article are included in the article's Creative Commons license, unless indicated otherwise in a credit line to the material. If materials are not included in the article's Creative Commons license and your intended use is not permitted by statutory regulation or exceeds the permitted use, you will need to obtain permission directly from the copyright holder.

To view a copy of this license, visit http://creativecommons.org/licenses/by-nc/4.0/.

(C) The Author(s) 2020. 